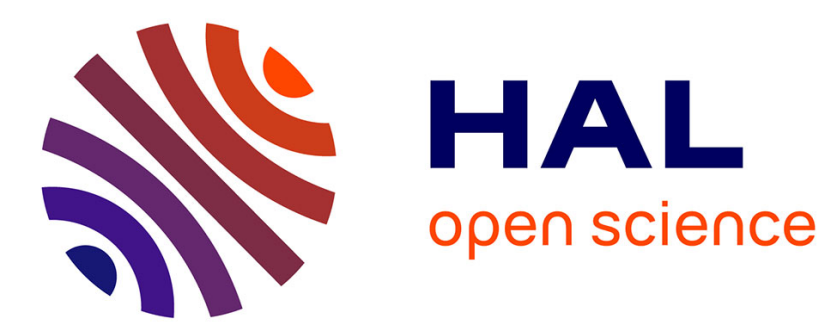

\title{
Dislocations et fautes d'empilement par microscopie ionique à champ
}

J. Gallot, D.A. Smith

\section{To cite this version:}

J. Gallot, D.A. Smith. Dislocations et fautes d'empilement par microscopie ionique à champ. Revue de Physique Appliquée, 1971, 6 (1), pp.11-18. 10.1051/rphysap:019710060101100 . jpa-00243496

\section{HAL Id: jpa-00243496 https://hal.science/jpa-00243496}

Submitted on 1 Jan 1971

HAL is a multi-disciplinary open access archive for the deposit and dissemination of scientific research documents, whether they are published or not. The documents may come from teaching and research institutions in France or abroad, or from public or private research centers.
L'archive ouverte pluridisciplinaire HAL, est destinée au dépôt et à la diffusion de documents scientifiques de niveau recherche, publiés ou non, émanant des établissements d'enseignement et de recherche français ou étrangers, des laboratoires publics ou privés. 


\title{
DISLOCATIONS ET FAUTES D'EMPILEMENT PAR MICROSCOPIE IONIQUE A CHAMP
}

\author{
J. GALLOT \\ Université de Rouen, Faculté des Sciences, Mont-Saint-Aignan, France
}

D. A. SMITH

Département de Métallurgie, Université, Oxford, Angleterre

(Reçu le 6 juillet 1970, révisé le 28 septembre 1970)

\begin{abstract}
Résumé. - L'image, donnée par microscopie ionique, d'une surface où émerge une dislocation, dépend principalement de la perturbation de cette surface le long de sa normale.

Les configurations de la surface pour des dislocations parfaites et imparfaites, des boucles, des fautes d'empilement et des dislocations dissociées, prédites théoriquement ont été corrélées avec les observations expérimentales dans les métaux cubiques faces centrées et cubiques centrés.

La théorie est aussi appliquée aux dislocations dans les joints de grains. On indique quelques limitations de la méthode et la validité des observations.

Abstract. - The field-ion image a surface in which a dislocation emerges depends principally on the perturbation of the surface along the local normal.

The surface configuration for single perfect and partial dislocations, loops, stacking faults and dissociated dislocations are deduced and correlated with experimental observations in face centred and body centred cubic metals.

The theory is also applied to dislocations in low angle and high angle grain boundaries. Some limitations of the methods of analysis and the validity of the observations are indicated.
\end{abstract}

Introduction. - Le microscope ionique à champ permet d'examiner la structure à l'échelle atomique d'une surface et par évaporations successives des couches d'atomes de cette surface, une investigation de l'échantillon massif est possible avec un pouvoir de résolution de 2 à $3 \AA$ Å. Il a été inventé par E. W. Müller en 1951 et depuis cette date, de nombreuses applications ont été mises au point. Elles appartiennent principalement à deux groupes distincts : le premier est l'étude des surfaces et croissance cristalline, le second comprend l'utilisation du microscope en physique du métal et métallurgie. Une revue en français sur les diverses applications de cette technique a été donnée par M. Drechsler [1]. Nous nous proposons de développer uniquement la partie intéressant les dislocations et fautes d'empilement en résumant d'abord les caractéristiques de la méthode qui interviendront dans le contraste obtenu.

I. Principe du microscope ionique à champ. L'échantillon est constitué par une pointe dont l'extrémité très fine peut être assimilée en première approximation à une demi-sphère d'un diamètre égal à quelques centaines d'Angströms. Il est porté à un potentiel positif par rapport à un écran fluorescent dans une enceinte dans laquelle le vide a été fait. Un gaz, appelé gaz imageant, est introduit sous faible pression dans l'enceinte et s'ionise préférentiellement aux endroits où le champ électrique est le plus intense, c'est-à-dire au voisinage immédiat de la surface hémisphérique. Ce phénomène fondamental mis en évidence par Inghram et Gomer [2] est l'ionisation par effet de champ. Il a été interprété comme le passage par effet tunnel d'un électron du gaz imageant vers le métal. Les ions produits vont former sur l'écran l'image de la modulation du champ électrique à la surface du spécimen. Expérimentalement, T. T. Tsong et E. W. Müller [3], en mesurant la distribution énergétique spatiale des ions formés, par la méthode des " potentiels retardés ", trouvent que la zone où l'ionisation a lieu se situe à une certaine distance critique $x_{\mathrm{c}}$ de la surface. Cette zone est très étroite. Jason [4], par cette même méthode, trouve un terme oscillant dans cette énergie en fonction de la distance $x$, à la surface de la pointe. Le contraste de l'image est donné par les fluctuations locales du champ au voisinage de la surface du spécimen. Ces fluctuations sont principalement dues au degré de protubérance des atomes à cette surface. Cette hypothèse permet d'expliquer la géométrie de l'image obtenue en considérant la pointe comme un empilement de sphères limité par la surface hémisphérique de l'échantillon. La comparaison d'une imagè ionique avec un empilement de sphères permet de voir que, au moins pour les plans compacts, seuls les atomes en 


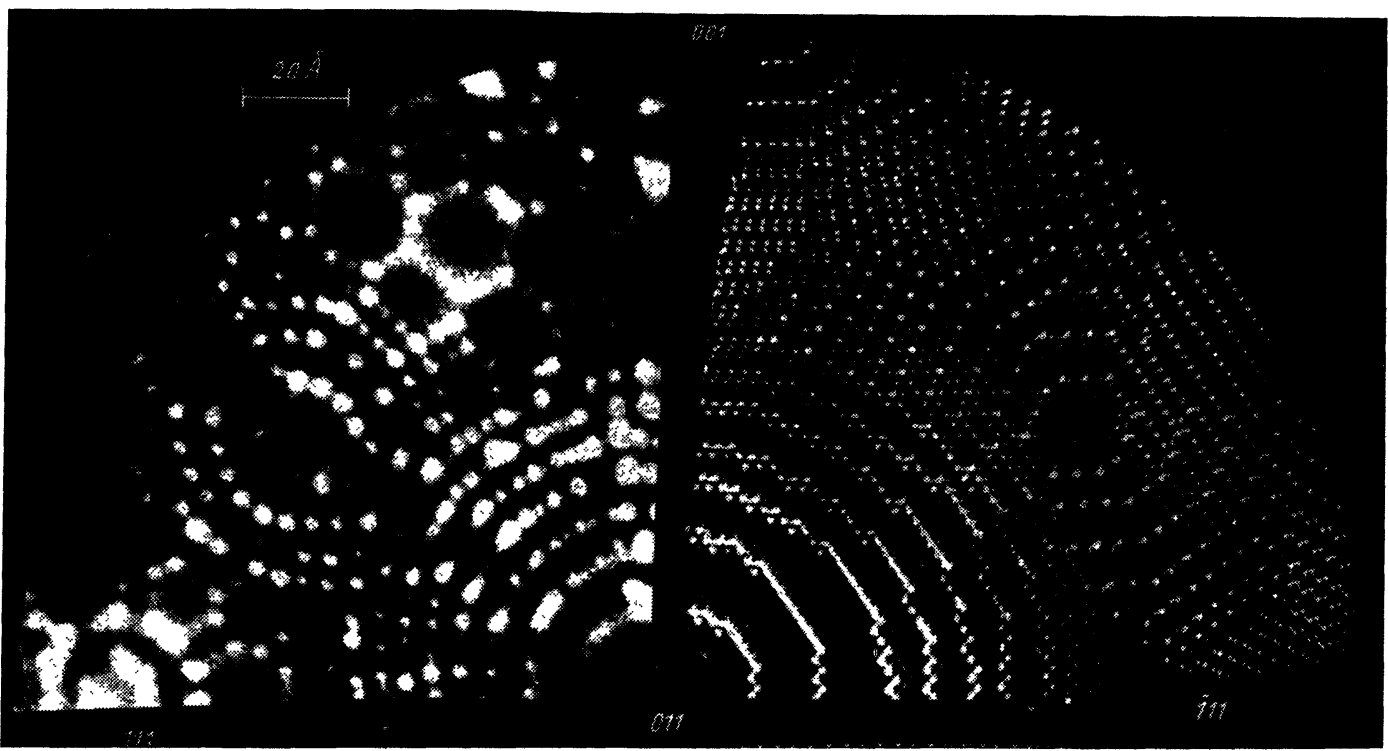

FIG. 1. - Modèle d'empilement de sphères. M. Drechsler, P. Wolf (1958) [5].

bordure d'un plan réticulaire donnent un pinceau d'ions (Fig. 1) (M. Drechsler et P. Wolf) [5].

Ce modèle suffit pour mettre sur pied une théorie géométrique des images données par les dislocations et fautes d'empilement dans les métaux purs. L'influence de la nature des atomes ou de la présence d'impuretés qui peuvent changer localement la distribution du champ électrique re sera pas prise en considération.

Un deuxième phénomène fondamental intervient dans la mise en œuvre de la microscopie ionique : l'évaporation par effet de champ E. W. Müller [6].

A partir d'un certain champ électrique $F_{\mathrm{E}}$ caractéristique pour un métal donné, l'évaporation des atomes protubérants se produit et ce phénomène peut se contrôler d'une façon très précise. En effet, en envoyant des pulses de tension au-dessus de la tension moyenne du spécimen, il est facile d'évaporer atome par atome la surface. Ceci permet l'investigation tridimensionnelle du spécimen. L'évaporation par effet de champ est aussi fondamentale dans la mise en œuvre de la méthode : l'interprétation des images obtenues est liée à leur régularité elle-même produite par celle de la surface à l'échelle atomique. Les pointes préparées par polissage électrolytique présentent des aspérités à cette échelle qui disparaissent préférentiellement par effet de champ jusqu'à l'obtention d'une forme d'équilibre. Enfin, l'évaporation par effet de champ limite l'emploi de la microscopie ionique aux métaux pour lesquels

$$
F_{\mathrm{E}}>F_{\mathrm{I}}
$$

$F_{\mathrm{E}}$ champ pour lequel l'évaporation par effet de champ se produit.

$F_{\text {I }}$ champ pour lequel l'ionisation par effet de champ a lieu.

C'est le cas des métaux réfractaires avec l'hélium. Il est possible d'employer des gaz dont l'énergie d'ionisation soit plus faible que celle de l'hélium, l'argon, l'hydrogène, ou des mélanges de ces différents gaz, sont généralement employés. Müller et al. [7] donnent des résultats avec des métaux moins réfractaires comme $\mathrm{Fe}, \mathrm{Au}, \mathrm{Co}, \mathrm{Ni}$, etc...

Il faut remarquer qu'au point de vue pratique, on doit distinguer deux cas : celui où la « résolution atomique » est atteinte (cas par exemple du platine Fig. 2)

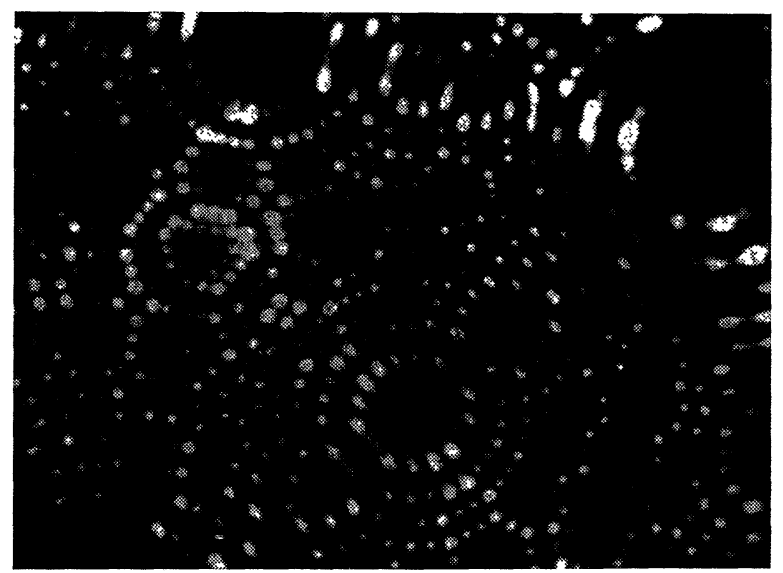

FIG. 2. - Platine à $21^{\circ} \mathrm{K}$ imagé à l'hélium. C. Martin, J. Gallot (non publié).

et celui où la « résolution atomique » n'est pas atteinte, mais où la présence de pôle bien céfini rend possible une analyse du contraste donné par dislocation et fautes d'empilement (cas du fer imagé par l'hydrogène Fig. 3).

Il faut remarquer que la résolution des atomes ne suffit pas pour étudier le cœur des dislocations qui nécessite une résolution inférieure à une distance interatomique de l'ordre de $0,2 \AA$. Nous verrons que celle-ci peut être obtenue dans une direction normale à l'échantillon. 


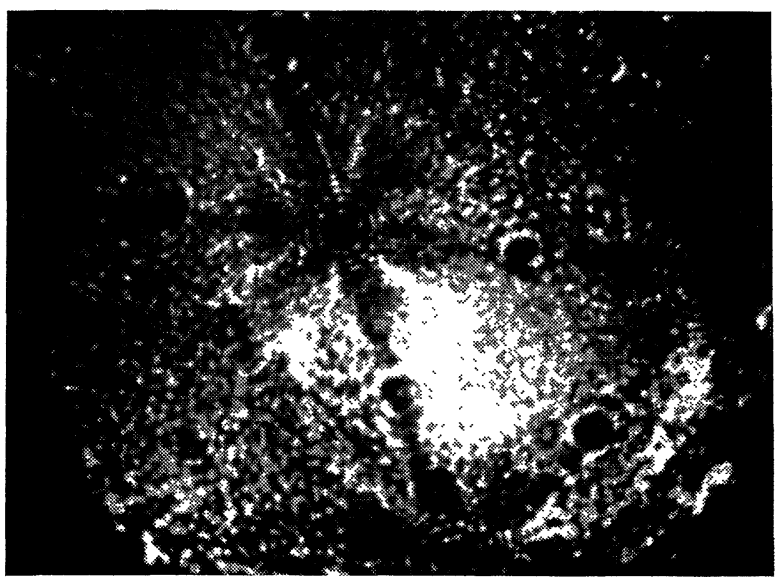

FIG. 3. - Fer à $78^{\circ} \mathrm{K}$ imagé à l'hydrogène. D. A. Smith, J. Gallot (1969) [12].

II. Théorie géométrique du contraste. - $a$, DisLoCATIONS PARFAITES. Pashley [8]. - L'image ionique est la projection de la répartition des atomes à la surface du spécimen. Une dislocation se manifestera par la structure de son intersection avec cette surface.

Le pôle parfait $(h k l)$ d'une micrographie ionique est constitué par une série de cercles concentriques formés par les plans atomiques de vecteur unitaire normal $\mathbf{n}$ qui coupent la surface de la pointe (Fig. 4a). Introduisons une dislocation du vecteur de Burgers b au centre de ce pôle figure $4 b$. Cette surface obtenue n'est pas stable et par évaporation par effet de champ, elle est transformée en une rampe hélicoïdale (Fig. 4c).

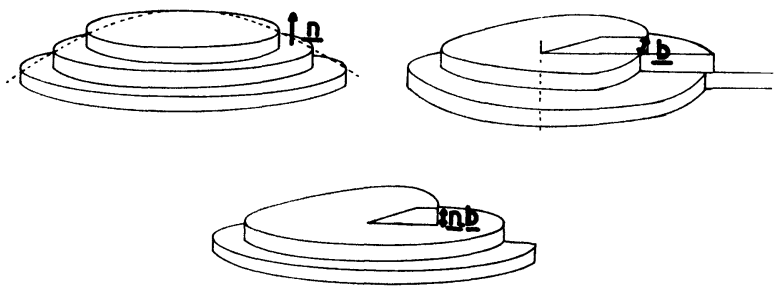

FIG. $4(a b c)$. - Dislocation parfaite $p=1$. D. A. Smith et al. (1968) [9].

Le circuit de Burgers peut être quelconque, en particulier il peut être choisi dans le plan de vecteur unitaire n. Dans le cas le plus général où $\mathbf{b} . \mathbf{n} \neq 0$, le pas de cette rampe est égal à n.b.

L'ionisation a lieu préférentiellement sur les bords du plan et l'image obtenue est une spirale (Fig. 5).

Dans le cas d'une dislocation parfaite, b est un vecteur du réseau et le produit n.b est égal au nombre entier de distances interplanaires.

$$
\mathbf{n} \cdot \mathbf{b}=p d_{h k l}
$$

soit $\mathbf{r}$ un vecteur du réseau réciproque

$$
\begin{aligned}
\mathbf{r}^{*} & =h \mathbf{a}^{*}+k \mathbf{b}^{*}+l \mathbf{c}^{*} \\
\mathbf{b} & =u \mathbf{a}+v \mathbf{b}+w \mathbf{c}
\end{aligned}
$$

REVUe DE PHYSIQUe APPLIQUÉE. - T. 6, No 1, MARS 1971

$$
\begin{aligned}
\mathbf{n} \cdot \mathbf{b} & =\frac{\mathbf{r}^{*} \cdot \mathbf{b}}{\left|\mathbf{r}^{*}\right|}=\frac{\left(h \mathbf{a}^{*}+k \mathbf{b}^{*}+l \mathbf{c}^{*}\right)(u \mathbf{a}+v \mathbf{b}+w \mathbf{c})}{\left|\mathbf{r}^{*}\right|} \\
\left|\mathbf{r}^{*}\right| & =\frac{1}{d_{h k l}} \\
\mathbf{n} \cdot \mathbf{b} & =(h u+k v+l w) d_{h k l}
\end{aligned}
$$

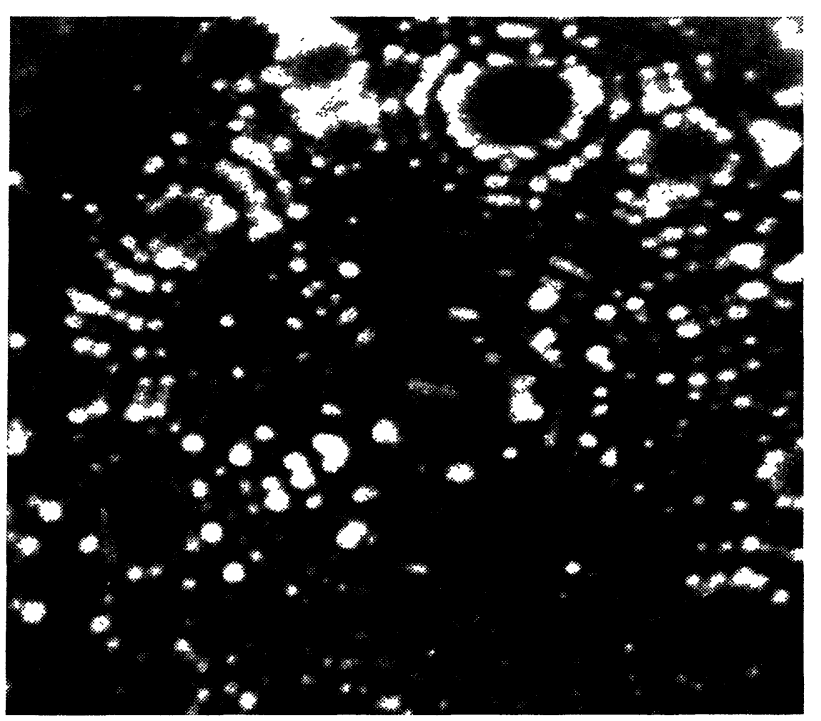

Fig. 5. - Dislocation parfaite dans du tungstène à $5 \%$ Rhénium. S. Collard, D. A. Smith (non publié).

$h k l$ sont choisis pour que l'espacement entre plans corresponde à la réalité. Par exemple, dans le cas d'un cubique faces centrées pour un pôle (110) on prendra

$$
h=2 \quad k=2 \quad l=0 .
$$

Cette équation s'applique aux réseaux contenant plus d'un atome par maille mais il est indispensable de travailler sur des plans cristallographiquement équivalents (dans le cas par exemple de l'hexagonal compact). La connaissance de la valeur numérique de

$$
p=h u+k v+l w
$$

permet de prévoir le contraste obtenu dans le cas d'une dislocation en tenant compte de la modification de la figure théorique prévue par l'évaporation par effet de champ qui fait tendre la surface de la pointe vers une surface idéale où aucun décrochement supérieur à une distance interatomique n'est possible. En appliquant ce principe au cas où $p=2$, on peut s'attendre à une spirale de pas égal à deux déplacements interplanaires (Fig. 6a). Cette structure de surface n'est pas stable et conduit par évaporation à la figure $6 b$. Celle-ci représente la micrographie ionique obtenue. La valeur de $p$ donne donc le nombre de spirales obtenues sur un pôle $h k l$ donné.

b. FAutes D'empilement. Smith et al. [9]. - Le même raisonnement que précédemment peut s'appliquer à une faute d'empilement. 

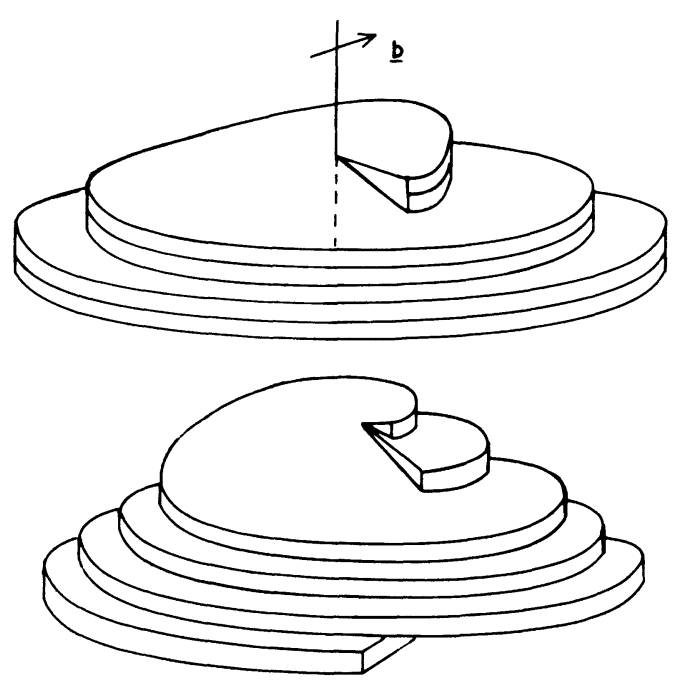

FIG. $6 a$ et $b$. - Dislocation parfaite $p=2$. D. A. Smith et al. (1968) [9].

Soient $\mathbf{R}$ le vecteur déplacement de la faute

$$
\mathbf{R}=u_{p} \mathbf{a}+v_{p} \mathbf{b}+w_{p} \mathbf{c}
$$

$u_{p} v_{p}$ et $w_{p}$ ne sont pas tous entiers.

s la normale unitaire au plan de la faute,

n la normale aux plans $h k l$ où la faute coupe la surface.

Dans le cas général $\mathbf{n} \wedge \mathbf{s} \neq 0$ un décrochement est formé à la ligne d'intersection de la faute et du plan $\mathbf{n}$.

La hauteur théorique du décrochement est

$$
\begin{gathered}
\mathbf{R} \cdot \mathbf{n}=q d_{h k l} \\
q=h u_{p}+k v_{p}+l w_{p}
\end{gathered}
$$

Le résultat obtenu est celui de la figure $8 b$, forme instable, qui par évaporation par effet de champ dans le cas où $q>1$ donne une hauteur de décrochement pratique égale à la partie non entière $q^{\prime}$ de $q$ (Fig. $7 c$, $d$ et $e$ ). Il faut remarquer que pendant une séquence d'évaporation enlevant successivement les différents plans, la hauteur du décrochement prend alternativement les valeurs

$$
q^{\prime},\left(1-q^{\prime}\right), q^{\prime},\left(1-q^{\prime}\right), \text { etc. }
$$

(a)

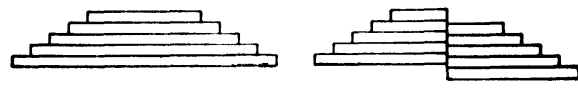

(c)

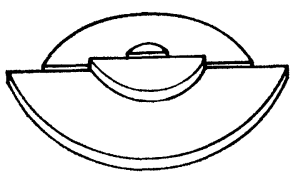

(d)

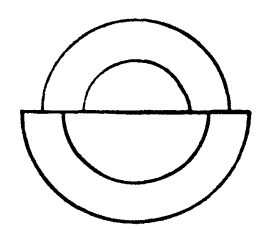

(e)
FIG. 7 (abcde). - Faute d'empilement. D. A. Smith et al. (1968) [9].
Dans le cas où $q$ est entier, la figure obtenue par évaporation ne présentera pas le décrochement caractéristique des fautes d'empilement. Un contraste pourra toutefois en résulter si le déplacement le long des rangées atomiques de l'intersection du plan de la faute avec le plan $h k l$ n'est pas égal à un nombre entier de distances interatomiques, le long de cette rangée. Ce déplacement est petit mais observable (Fig. 8).

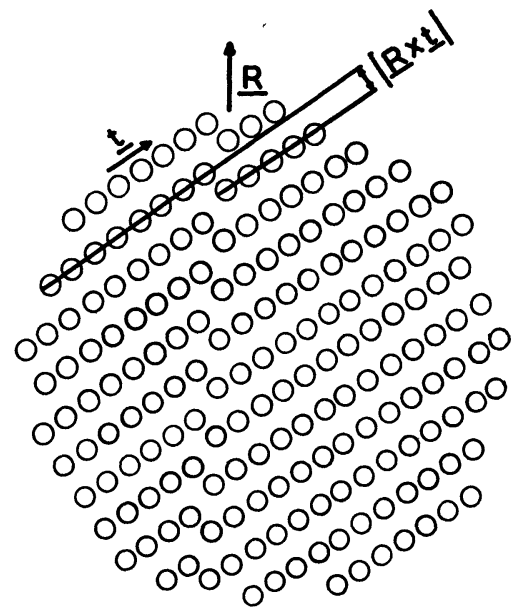

FIG. 8. - Faute d'empilement $\mathbf{R}$ dans le plan de la faute.

c. Dislocations Partielles. - Une dislocation partielle borde une faute d'empilement. Son intersection avec la surface de la pointe produira les effets dus"à la faute et à la dislocation

$$
\mathbf{b}_{p}=\mathbf{R}
$$

$\mathbf{b}_{p}$ vecteur de Burgers de la dislocation partielle.

Comme précédemment, le contraste dépend de $q$.

$$
0<|q|<1
$$

Le pas de la spirale est plus petit qu'une distance interplanaire (Fig. 9).

$$
|q|=\text { entier . }
$$

On obtient comme dans le cas d'une dislocation parfaite $q$ spirales mais un contraste peut exister, dû au déplacement le long des rangées atomiques qui bordent la faute comme dans le cas d'une faute d'empilement pour laquelle $\mathbf{R} \cdot \mathbf{n}=$ entier.

$$
|q|>1 \text { mais non entier . }
$$

On obtient une spirale multiple dont le pas n'est pas égal à un nombre entier de distance interplanaire.

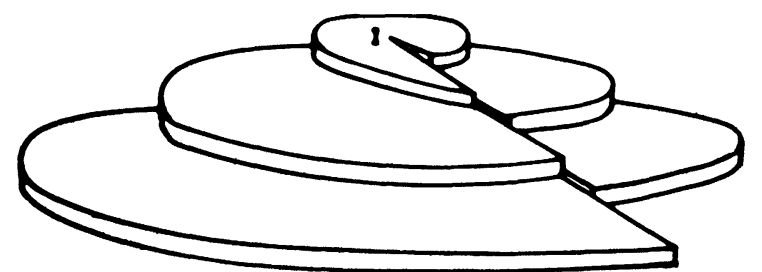

FIG. 9. - Dislocation imparfaite. D. A. Smith et al. (1968) [9]. 
III. Applications de la théorie. - La connaissance de $p$ ou de $q$ pour un défaut déterminé permet de prévoir le contraste qu'il donnera sur une micrographie ionique. Il est utile de prendre une convention de signe pour le calcul de $p$ et $q$. En effet deux dislocations de vecteurs de Burgers opposés, émergeant en un même pôle, apparaîtront sous la forme de deux spirales de sens opposé.

La ligne de dislocation peut être déterminée par évaporations successives des couches d'atomes, en suivant la trace de son intersection avec la surface. Nous orientons arbitrairement la ligne de dislocation à cette intersection, soit $\mathbf{l}$ son vecteur unitaire choisi tel que I.n $>0$. Le sens de la spirale dépend alors du signe de b.n.

$$
\begin{aligned}
& \text { b.n }<0 \text { spirale de sens direct. } \\
& \text { b.n }>0 \text { spirale de sens indirect. }
\end{aligned}
$$

a. Dipoles ET BOUCles DE Dislocations. - Ces deux systèmes présenteront un contraste équivalent correspondant à deux dislocations de vecteur de Burgers $b_{1}=-b_{2}$ opposés. Emergeant dans un même pôle, les spirales seront de sens contraire $p_{1}=-p_{2}$. Tout cercle d'atomes entourant les deux dislocations et correspondant à $\mathbf{b}=\mathbf{b}_{1}+\mathbf{b}_{2}=0$ sera parfait (Fig. 10).

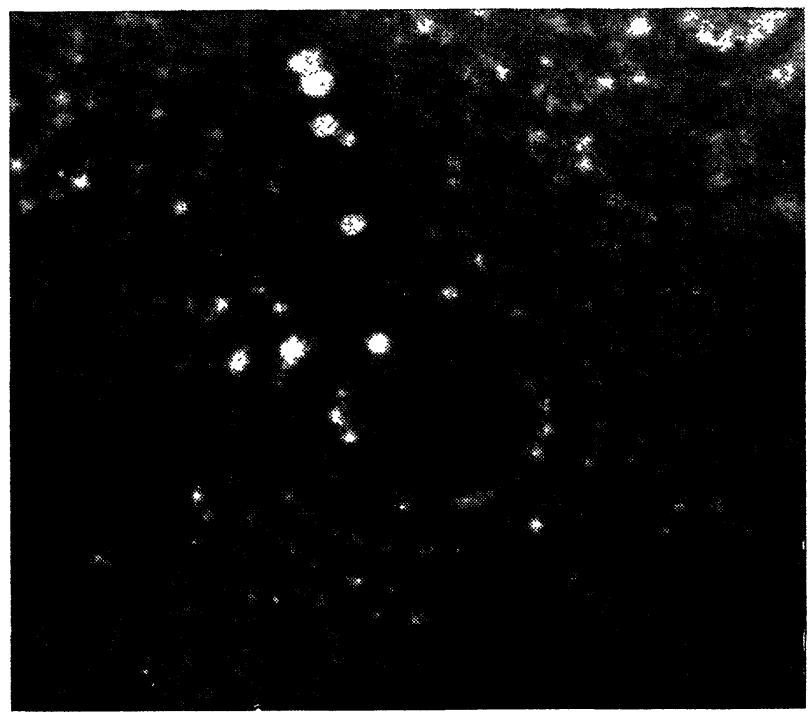

Fig. 10. - Boucle de dislocations dans l'iridium. M. A. Fortes et al. (1968) [10].

On distingue un dipôle d'une boucle par l'examen d'une séquence d'évaporation.

$b$. Sous-JOINTS. Fortes et al. [10]. - Il est possible de décrire un joint de grain par une série de dislocations dont la distribution est donnée par la relation de Frank

$$
\mathbf{S}=(\mathbf{U} \wedge \mathbf{V}) \theta
$$

$\mathbf{S}$ vecteur de Burgers,

$\mathbf{U}$ axe de désorientation,

$\mathbf{V}$ vecteur du plan de joint,

$\theta$ rotation relative des deux grains.
Dans ce cas, un cercle d'atomes entourera éventuellement plusieurs dislocations et le contraste obtenu dépend de la somme de leurs vecteurs de Burgers. Des spirales de multiplicité inattendue peuvent être produites.

L'évaporation par effet de champ permet par l'analyse de la trace du joint sur l'image de déterminer sa place.

On doit remarquer que si l'angle $\theta$ est petit, l'espacement de dislocations est grand par rapport au champ du microscope et une seule dislocation peut être éventuellement seule visible.

Des spirales se forment souvent dans les joints de grains de forte désorientation mais ce phénomène n'est pas toujours dû aux dislocations. Dans certains cas, c'est probable : lorsque dans un plan ( $h k l)$ la désorientation est au bout d'un axe [hkl] les spirales existantes sont dues aux dislocations. La micrographie ionique montre une macle $\left(70^{\circ} 32^{\prime}\right)$ au bout de [110] avec des spirales dues à des dislocations (Fig. 11). La figure 12 montre le même échantillon sur microscope électronique.
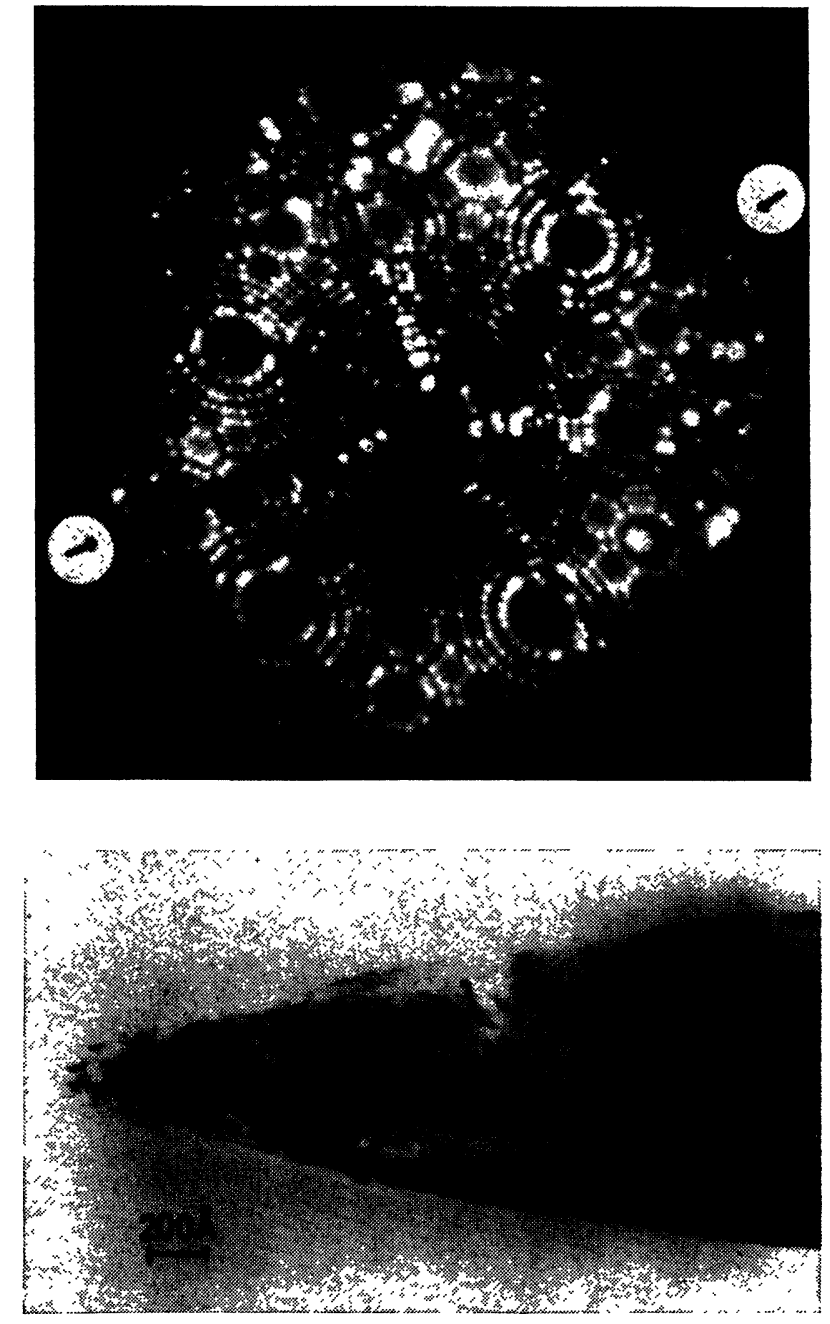

Fig. 11 et 12. - Macle dans du tungstène par microscopie ionique et électronique. B. Loberg, H. Norden, D. A. Smith (sous presse) [18] 
c. Dissociation DE Dislocation. - Il existe de nombreux contrastes possibles donnés par une dislocation parfaite dissociée. Mais dans tous les cas, le contraste peut être analysé en fonction des paramètres $p$ pour la dislocation parfaite et $q$ pour les dislocations partielles.

Prenons comme exemple la réaction

$$
\begin{aligned}
& \frac{a}{2}[101]_{(11 \overline{1})} \rightarrow \frac{a}{6}[2 \overline{1} 1]_{(11 \overline{1})}+\frac{a}{6}[112]_{(11 \overline{1})} \\
& p=1 \quad q=\frac{1}{3} \quad q=\frac{2}{3} .
\end{aligned}
$$

A partir des valeurs de $p$ et $q$, il est possible de prévoir le contraste obtenu. Les cercles d'un pôle entourant les deux partielles formeront une simple spirale $(p=1)$ correspondant à la dislocation totale mère. Si on considère les cercles entourant seulement une des dislocations partielles, en $H$ par exemple $q=\frac{1}{3}$ et la spirale ne se referme pas (Fig. 13).

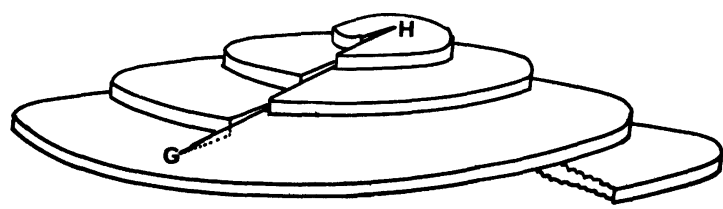

Fig. 13. - Dislocation dissociée. D. A. Smith et al. [9].

Dans certains cas, il est impossible de déterminer le type de défauts sans examiner une séquence d'évaporation qui fait passer la dislocation successivement à travers plusieurs pôles. C'est le cas d'un dipôle et d'une boucle qui donne le même contraste.

C'est aussi le cas d'une boucle imparfaite et d'une dislocation dissociée dont le vecteur de Burgers total est tel que $p=0$ (Fig. 14).

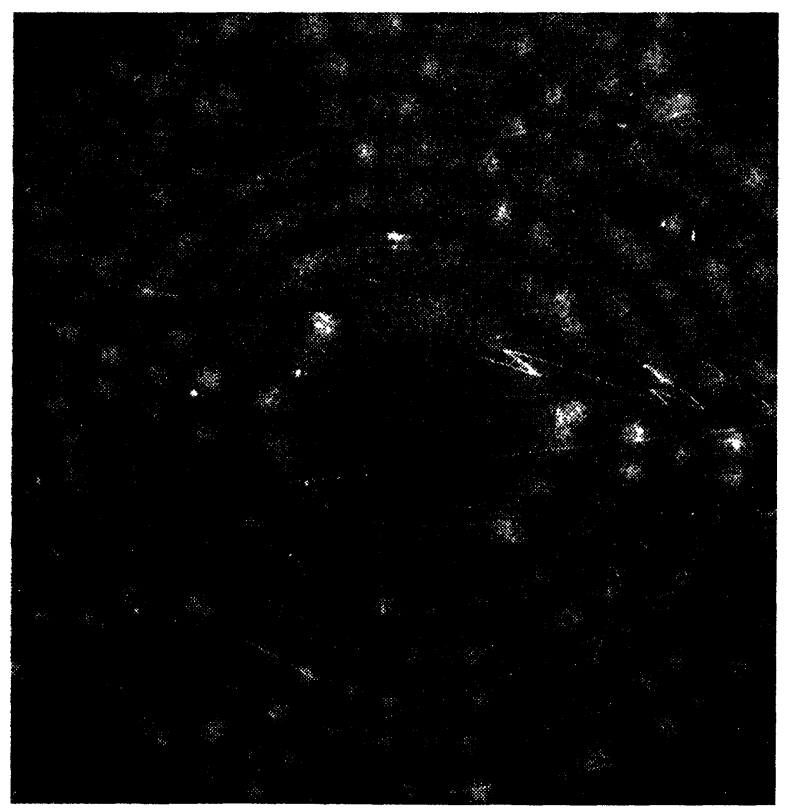

FIG. 14. - Boucle imparfaite dans du Tungstène. C. Martin, J. Gallot (non publié).
IV. Limitation et précision de l'analyse. - Nous venons de montrer que la théorie permet de prévoir le contraste donné par un système quelconque de dislocations et de fautes d'empilement. L'inverse est beaucoup plus difficile; trouver sans ambiguïté à quel type de défaut correspond un contraste trouvé expérimentalement sur une micrographie ionique n'est pas toujours possible et conduit souvent à plusieurs solutions.

a. Détermination DU VeCteur DE Burgers. - Il faut calculer les différentes valeurs possibles de $p$ correspondant au nombre de spirales données par une dislocation de vecteur de Burgers b émergeant en différents pôles.

Sauf pour quelques cas particuliers (Fig. 15), où la solution $p=3$ pour le pôle (331) est unique : $b=a / 2$ [110], plusieurs vecteurs de Burgers peuvent donner le même nombre de spirales.

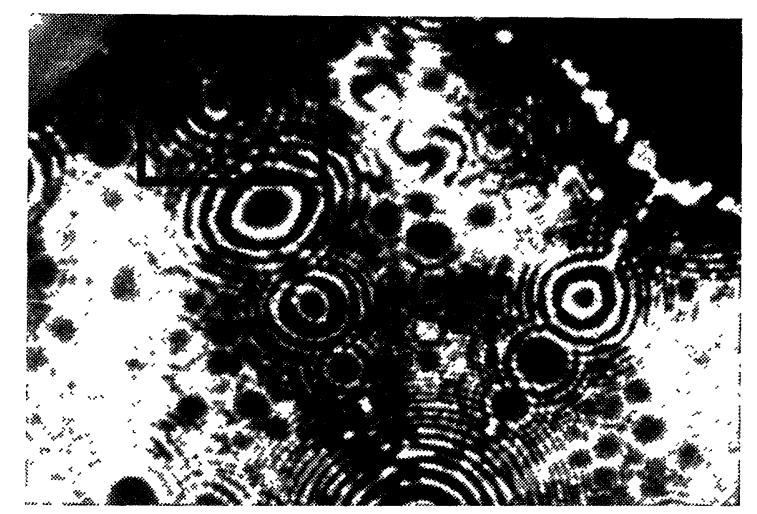

FIG. 15. - Triple spirale dans l'iridium. T. F. Page (1970) [19].

Plusieurs méthodes permettent de lever cette indétermination en prenant plusieurs valeurs de $h k l$ pour une même dislocation.

- Soit par évaporation par effet de champ:1a dislocation coupe successivement la surface aux plans $h_{1} k_{1} l_{1}$ et $h_{2} k_{2} l_{2}$.

- Soit en considérant la dislocation comme appartenant à deux pôles différents $\left(h_{1} k_{1} l_{1}\right)\left(h_{2} k_{2} l_{2}\right)$ et en observant les effets de la dislocation sur chacun de ces pôles (Fig. 16).

Un certain nombre de précautions sont à prendre dans l'interprétation des spirales multiples. En effet, celles-ci peuvent être dues non à une dislocation unique mais à plusieurs dislocations émergeant dans un même pôle. (On additionne alors les valeurs de $p$.) Il est préférable dans ces cas d'étudier la variation de $p$ suivant les différents pôles. L'exemple des dislocations $\mathbf{b}=\langle 110\rangle$ dans le fer pur a été longuement discuté.

En effet deux dislocations de vecteur $a / 2$ [111] et $a / 2[1 \overline{1}]$ peuvent donner le même contraste. Cette discussion a surtout porté sur la stabilité d'un tel système de deux dislocations assez rapprochées puisqu'elles émergent dans un même pôle. L'évaporation 


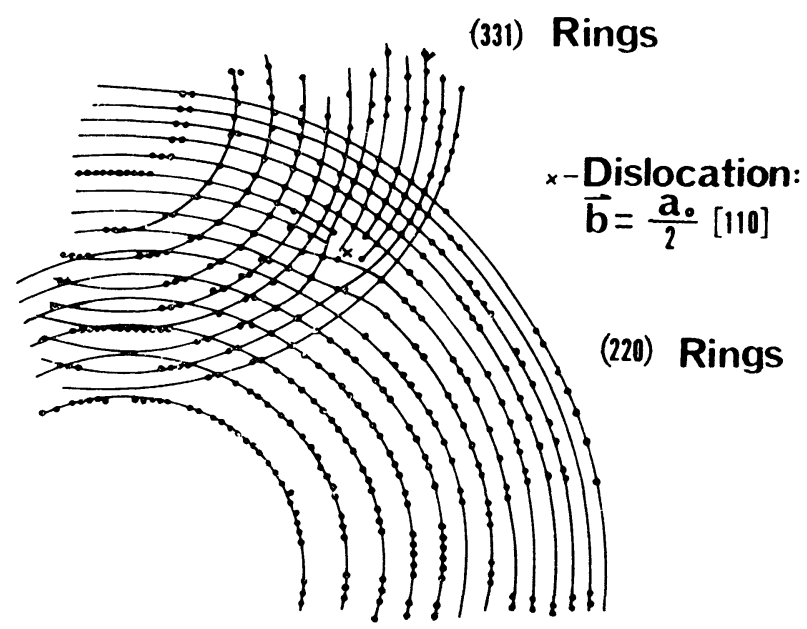

FIG. 16. - Simulation à l'ordinateur. R. C. Sanwald, J. J. Hren (1968) [20].

par effet de champ permet de distinguer les deux cas avec une précision de $20 \AA$ : dans le cas où deux dislocations existent, le rebord d'un plan doit passer entre les deux extrémités de la spirale (Fig. 17).
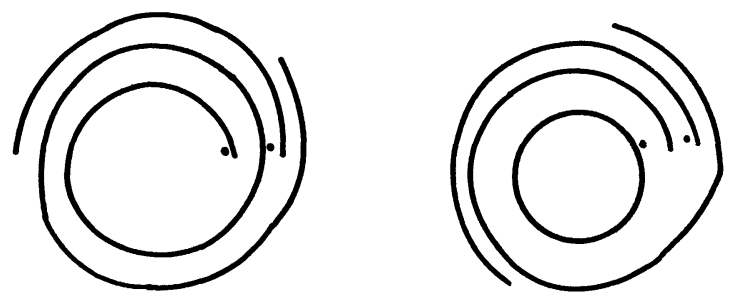

FIG. 17. - Double spirale formée de deux spirales simples.

$b$. DÉTERmination du PlAN DE LA FAUTE. - Diverses solutions ont été proposées pour relier la structure de la surface de la pointe à l'image obtenue. L'hypothèse la plus généralement utilisée est celle de Drechsler et Wolf [5] qui assimilent l'image à une projection stéréographique de la répartition des atomes à la surface de la pointe. Cela a permis d'indexer les différents pôles mais le problème de la détermination absolue d'un plan quelconque demande une précision énorme dans la connaissance des différents paramètres qui déterminent l'image de la faute.

Le premier facteur qui intervient est la forme de la pointe, la faute ne se manifeste que par son intersection avec la surface. Le deuxième facteur est la trajectoire des ions depuis cette intersection jusqu'à l'écran. Ces deux facteurs sont liés et difficiles à traiter théoriquement avec précision, la trajectoire des ions au voisinage immédiat de la pointe a un rôle prépondérant.

Les différentes solutions utilisées sont empiriques et tirées de résultats expérimentaux.

- Forme de la pointe. - La forme est différente suivant les différents plans atomiques comme le montrent les profils des pointes obtenues par microscopie électronique (Fig. 18). Il est possible de mesurer

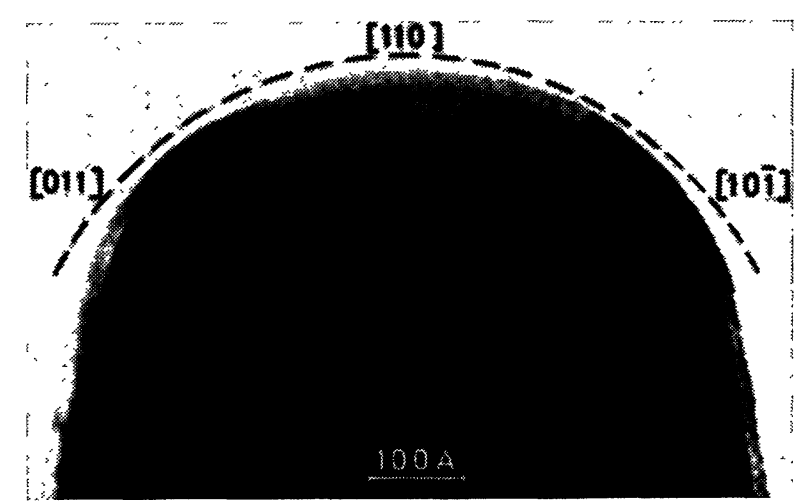

FIG. 18. - Profil de pointe par microscopie électronique. B. Loberg, H. Norden (1969) [15].

ce rayon de courbure local directement sur les micrographies ioniques à l'aide de l'espacement des anneaux.

- Type de projection. - Comme le rayon de courbure n'est pas constant d'un point à un autre des spécimens, le pôle de projection ne sera pas le même pour tous les points de l'image. Pour les défauts de petites dimensions (boucle fautes d'empilement) on considère le rayon local et on assimile la projection à une projiction stéréographique.

c. Ligne DE dislocation. - Comme la faute planaire, la ligne de dislocation peut être déterminée par évaporation du spécimen par plans successifs en suivant la trace de l'interaction avec la surface, Smith Bowkett [11].

Seule cette détermination permettra de mettre en évidence le caractère vis de la dislocation. On doit en effet remarquer que n.b n'est pas en général la composante vis.

d. Dislocation DISSOCIÉE DANS LES MÉTAUX CUBIQUES CENTRÉS. - Des fautes d'empilement ont été trouvées par microscopie ionique dans les métaux cubiques centrés (Fig. 4).

Ces fautes ont généralement été interprétées comme une dissociation de dislocations (Smith-Gallot, [12] est-ce la seule solution ? et quels sont les défauts qui, coupant une surface, donnent un déplacement normal à celle-ci d'un type identique à celui donné par une faute d'empilement ?

Les précipités semi-cohérents, les micro-macles postulées par Sleeswyk et les dislocations dissociées donnant une faute intrinsèque donnent un tel contraste.

Les défauts trouvés dans les métaux cubiques faces centrées ont les caractéristiques suivantes;

- Décrochement associé à une faute ;

- Absence de variation de contraste ;

- Spirale parfaite entourant le défaut.

L'observation ne montre pasfle contraste habituel d'une seconde phase : région de contraste différente et d'autre part, les matériaux examinés étaient de haute pureté, il est improbable que ces défauts soient des précipités. 


\section{- Macle}

La présence de la spirale entourant le défaut élimine ce cas.

- Germe de macle.

La figure 19 montre le contraste prévu pour une dislocation dissociée suivant Sleeswyk [13]. Il est caractérisé par un défaut se formant sur trois couches et par le fait que les anneaux ne présentent aucun déplacement quand une dislocation n'est pas entourée.

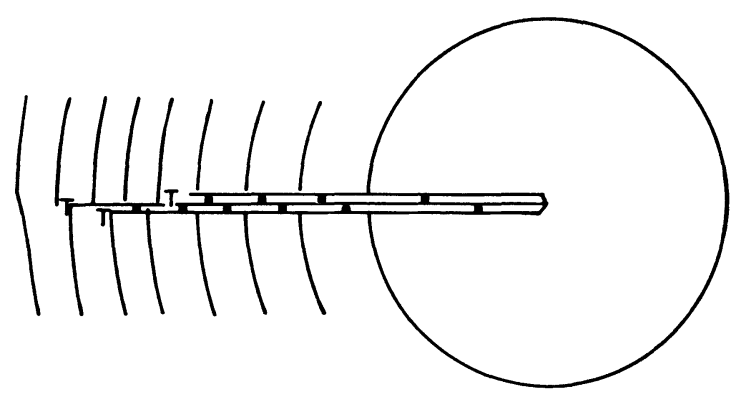

FIG. 19. - Contraste prévu pour un germe de macle.

Les défauts précédents ne sont donc pas des niveaux de macles à moins qu'une dislocation soit située à la tête de la faute.

Vitek [14] a montré par calcul que les défauts intrinsèques ne sont pas stables dans le métal massif. Mais le spécimen examiné n'est pas massif et la présence de grandes contraintes peuvent changer l'énergie de faute et la largeur des rubans.

Conclusion. - La thésrie géométrique du contraste permet d'aborder avec une certaine réussite l'analyse des dislocations et fautes d'empilement présentes sur les micrographies ioniques. Une question doit être posée : quelle est la relation entre les propriétés méca- niques de la pointe et celles du même métal à l'état massif. Deux phénomènes principaux vont tendre à les différencier :

- effet de la surface ;

- effet des contraintes dues au champ électrique.

Ces deux phénomènes peuvent modifier la distribution des dislocations mobiles, les micrographies électroniques (Loberg) [15] effectuées sur un échantillon avant et après introduction dans le microscope ionique montrent qu'il ya diminution de la densité des dislocations. Cette diminution a déjà probablement lieu pendant la préparation des lames minces. Mais, ce fait est en compétition avec la germination homogène de dislocation au moment où le champ électrique est le plus élevé c'est-à-dire au moment de l'évaporation par effet de champ (Fortes Ralph) [16].

Les effets de la surface et les effets de contraintes dues au champ électrique ne se font pas seulement sentir sur la distribution des dislocations.

La largeur des rubans de faute est beaucoup plus élevée que prévue théoriquement et ceci est certainement une conséquence des conditions expérimentales d'examen de l'échantillon. Sous l'action du champ électrique la distance entre premiers voisins augmente (Rendulic Müller) [17] et cette augmentatuon peut conduire à stabiliser des défauts instables dans les conditions normales.

La théorie géométrique du contraste est une première étape dans la compréhension des phénomènes mécaniques qui se passent dans un échantillon de microscopie ionique. Et si la relation entre les phénomènes étudiés et les propriétés d'un échantillon massif présente certaines difficultés, le comportement des dislocations sous l'action de ces contraintes est en soi intéressant.

\section{Bibliographie}

[1] Drechsler (M.), Revue de Physique Appliquée, 1968, $3,331$.

[2] Inghram (M. G.), Gomer (R.), J. Chem. Phys., 1954, 22, 1279.

[3] Tsong (T. T.), Müller (E. W.), J. Chem. Phys. 1964, 41, 3279.

[4] Jason (A. J.), Phys. Rev., 1967, 156, 156.

[5] Drechsler (M.), Wolf (P.), IV Int. Kongr. Elek. Mikr (1958), 1, 835, Springer Verlag Berlin.

[6] Müller (E. W.), Phys. Rev., 1956, 102, 618.

[7] Müller (E. W.), Nakamura (S.), Nishikawa (S.), McLan, J. Appl. Phys., 1965, 36, 2496.

[8] Pashley (D. W.), Rept. Prog. Phys., 28, 291.

[9] Smith (D. A.), Fortes (M. A.), Kelly (A.), Ralph (B.), Phil. Mag., 1968, 17, 1065.

[10] Fortes (M. A.), Smith (D. A.), Ralph (B.), Phil. Mag., 1968 17, 169.
[11] Sмith (D. A.), Bowkett (K. M.), Phil. Mag., 1968, 18, 1219

[12] Smith (D. A.), Gallot (J.), Metal Science Journal, $1969,3,79$.

[13] SleeswyK (A. W.), Phil. Mag., 1963, 8, 1467.

[14] ViteK (V.), Phil. Mag., 1968, 773.

[15] Loberg (B.), Norden (H.), Arkiv for Fysik, 1969, 39, 383.

[16] Fortes (M.), Ralph (B.), Phil. Mag., 1968, 18, 787.

[17] Rendulic (K. D.), Müller (E. W.), J. Appl. Phys., 1967, 38, 2070.

[18] Loberg (B.), NoRden (H.), SMIth (D. A.), Arkiv for Fysik (à paraître).

[19] Page (T. F.), Phil. D. Thesis (1970), Cambridge University.

[20] Sanwald (R. C.), Hren (J. J.), Surface Science, 1968, 9, 257. 